\title{
Fungal diversity in wounded stems of Aquilaria malaccensis.
}

\begin{abstract}
Aquilaria malaccensis is a tropical tree which produces agarwood in its trunk often after being wounded and attacked by pathogens or insects. Fungi are generally viewed as the main microbial component responsible for agarwood formation. In this study, isolation of fungi from agarwood in damaged trees was carried out. Culture morphology and microscopic characteristics plus PCR amplification of the internal transcribed spacer (ITS) region from the fungal isolates as well as wood samples, were used to identify the fungal community composition of wounded A. malaccensis trees from a natural forest in West Malaysia. Conventional culture methodology revealed Cunninghamella, Curvularia, Fusarium and Trichoderma species as members of the agarwood community. Analysis of genomic DNA confirmed the identifications. When wood samples were used directly in PCR, an additional Lasiodiplodia species was identified. Neighbor-joining trees were constructed to examine the relationships between the isolates sequence data and reference sequences in GenBank. Five distinct clades resulted, supported with high bootstrap values, indicating the presence of five distinct taxa. The wounded trunks of $\mathrm{A}$. malaccensis in the natural environment harbor multiple fungal taxa that exist in a complex system as a whole or in succession leading to agarwood production in the tree trunk.
\end{abstract}

Keyword: Agarwood; Aquilaria malaccensi 